\title{
Nab-paclitaxel is effective against intrahepatic cholangiocarcinoma via disruption of desmoplastic stroma
}

\author{
PETER MU-HSIN CHANG ${ }^{1,2}$, CHI-TUNG CHENG $^{3}$, REN-CHIN WU $^{4}$, YI-HSIU CHUNG ${ }^{5}$, KUN-CHUN CHIANG $^{3}$, \\ TA-SEN YEH ${ }^{3}$, CHUN-YU LIU ${ }^{1,2}$, MING-HAN CHEN ${ }^{2}$, MING-HUANG CHEN ${ }^{1,2^{*}}$ and CHUN-NAN YEH ${ }^{3 *}$ \\ ${ }^{1}$ Department of Oncology, Taipei Veterans General Hospital; ${ }^{2}$ School of Medicine, National Yang-Ming University, \\ Taipei $112 ;{ }^{3}$ Department of Surgery and Liver Research Center; ${ }^{4}$ Department of Pathology; \\ ${ }^{5}$ Center for Advanced Molecular Imaging and Translation, Chang Gung Memorial Hospital, Taoyuan 333, Taiwan, R.O.C.
}

Received January 19, 2017; Accepted February 2, 2018

DOI: $10.3892 / \mathrm{ol} .2018 .8690$

\begin{abstract}
Intrahepatic cholangiocarcinoma (IH-CCA) is the second predominant hepatic malignancy worldwide. However, effective treatment strategies for IH-CCA have not yet been developed. Nab-paclitaxel may be an effective drug against IH-CCA, a type of desmoid-like tumor, and its antitumor effects may be attributable to its ability to disrupt the cancer-associated fibroblasts. In the present study, MTT and Annexin-V apoptosis detection kits were used to evaluate the efficacy of paclitaxel and nab-paclitaxel against human cholangiocarcinoma KKU-100 and KKU-213 cell lines. A rat model of thioacetamide-induced spontaneous desmoplastic IH-CCA was used to compare the treatment response of four different drug regimens: Control, paclitaxel, nab-paclitaxel and gemcitabine/oxaliplatin. Positron emission tomography and immunofluorescence analysis were used to measure the tumor volume and to study the resected tumor, respectively. In vitro, paclitaxel and nab-paclitaxel induced anti-proliferative effects in KKU-100 and KKU-M213 cells. With regards to the treatment regimes, only nab-paclitaxel and gemcitabine/oxaliplatin induced antitumor effects in the rat model of thioacetamide-induced IH-CCA. The immunofluorescence study indicated that nab-paclitaxel was more efficient in disrupting cancer-associated fibroblasts than paclitaxel. In
\end{abstract}

Correspondence to: Dr Chun-Nan Yeh, Department of Surgery and Liver Research Center, Chang Gung Memorial Hospital, 5 Fuhsing Street, Taoyuan 333, Taiwan, R.O.C.

E-mail: yehchunnan@gmail.com

Dr Ming-Huang Chen, Department of Oncology, Taipei Veterans General Hospital, National Yang-Ming University, 201, Section 2, Shih-Pai Road, Taipei 112, Taiwan, R.O.C.

E-mail: mhchen9@gmail.com

*Contributed equally

Key words: cholangiocarcinoma, cancer-associated fibroblast, nab-paclitaxel, desmoplastic, $\alpha$-smooth muscle actin conclusion, nab-paclitaxel is effective against IH-CCA owing to its ability to markedly disrupt the desmoplastic stroma.

\section{Introduction}

Intrahepatic cholangiocarcinoma (IH-CCA) is a malignant epithelial tumor arising from hepatic parenchyma in the biliary tracts. It is the second most prevalent primary hepatic tumor worldwide with a progressive increase in incidence and mortality rates in recent years (1-3). At present, complete surgical resection is the only possible curative therapy for patients with IH-CCA. For unresectable IH-CCA, palliative chemotherapy with cisplatin and gemcitabine is the standard first-line treatment. However, the response to such therapy remains limited (4). Since no effective drug is available for the treatment of refractory CCA $(5,6)$, novel therapeutic drugs require urgent development.

A possible explanation for the multi-drug resistance observed in patients with IH-CCA is the 'desmoid-like' pattern of IH-CCA in the liver parenchyma. IH-CCA is well known for its desmoplastic and hypoplastic stroma, which are both predictive factors for poor drug penetration and response (7). Recently, $\alpha$-smooth muscle actin ( $\alpha$-SMA) expressed by cancer-associated fibroblasts (CAFs), was revealed to have a major role in altering the tumor microenvironment by increasing the production of extracellular matrix proteins, cytokines and growth factors that interact with CCA, thereby enhancing the aggressive behavior and therapeutic resistance of the tumor (8).

Several CAF-associated liver parenchyma diseases have been linked to the risk of developing CCA. For example, primary sclerosing cholangitis, a chronic inflammatory disease of the bile duct characterized by fibrosis in the biliary tract, has been identified as a predisposing factor for IH-CCA. Immunohistochemical staining has revealed the enhanced expression of $\alpha$-SMA in the biliary stroma of these patients (7). The presence of desmoplasia is also indicative of the existence of a close association between mammographic density and breast cancer (9).

Nab-paclitaxel, also known as nanoparticle albumin-bound paclitaxel, is designed to deliver the paclitaxel compound without the complications induced by chemical solvents. A 
follow-up study reported stroma disrupting effects of nab-paclitaxel in pancreatic cancer (10), which were confirmed later in a phase III MPACT study that suggested that nab-paclitaxel with gemcitabine is more effective than gemcitabine alone in the treatment of pancreatic cancer (11). However, the role of nab-paclitaxel in treating IH-CCA has not been investigated despite the fact that IH-CCA is also a desmoplastic tumor.

The present study we initially investigated the inhibitory effects of paclitaxel and nab-paclitaxel in different CCA cell lines. Subsequently, a toxin-induced IH-CCA rat model established in our laboratory was used to evaluate the in vivo antitumor activity of the standard gemcitabine/oxaliplatin, paclitaxel and nab-paclitaxel treatments. Compared with paclitaxel, nab-paclitaxel demonstrated increased effectiveness in reducing in vivo tumor formation by disrupting the desmoplastic stroma.

\section{Materials and methods}

Cell culture. The CCA KKU-M213 and KKU-100 cell lines were obtained from the Japanese Collection of Research Bioresources Cell Bank (JCRB; Osaka, Japan). KKU-213 and KKU-100 cells were cultured in Dulbecco's modified Eagle's medium (DMEM; Gibco; Thermo Fisher Scientific, Inc., Waltham, MA, USA), supplemented with $10 \%$ heat-inactivated fetal bovine serum (FBS; GE Healthcare Life Sciences, Little Chalfont, UK), $100 \mu \mathrm{g} / \mathrm{ml}$ streptomycin, $100 \mu \mathrm{g} / \mathrm{ml}$ penicillin and $2 \mathrm{mM}$ L-glutamine (Invitrogen; Thermo Fisher Scientific, Inc.) in a humidified atmosphere containing $5 \% \mathrm{CO}_{2}$ at $37^{\circ} \mathrm{C}$.

Cell viability measurements. Cell viability was determined using an MTT cell viability assay kit (Trevigen, Inc., Gaithersburg, MD, USA), according to manufacturer's protocols. The cells were seeded at a density of 2,000 cells/100 $\mu \mathrm{l}$ culture medium/well in 96-well microplates. At $24 \mathrm{~h}$ after seeding, the cells were treated with $0,5,10,20,40,80,160$ or $320 \mathrm{nM}$ paclitaxel or nab-paclitaxel dissolved in dimethyl sulfoxide (DMSO) or DMEM medium containing DMSO and $10 \%$ heat-inactivated fetal bovine serum (FBS; GE Healthcare Life Sciences), $100 \mu \mathrm{g} / \mathrm{ml}$ streptomycin, $100 \mu \mathrm{g} / \mathrm{ml}$ penicillin and $2 \mathrm{mM}$ L-glutamine (Invitrogen; Thermo Fisher Scientific, Inc.) for $72 \mathrm{~h}$ in a humidified atmosphere containing $5 \% \mathrm{CO}_{2}$ at $37^{\circ} \mathrm{C}$. Subsequently, the cells were incubated in the medium containing MTT for $4 \mathrm{~h}$ and were lysed with DMSO, prior to the optical density of the resulting supernatant being measured at $570 \mathrm{~nm}$ using a microplate reader (Spectral Max 250; Molecular Devices, LLC, Sunnyvale, CA, USA).

Apoptotic cell death. Apoptosis was measured using a FITC Annexin V apoptosis detection kit (BD Biosciences, Franklin Lakes, NJ, USA), according to manufacturer's instructions. After a 24-h incubation, cells were treated with DMSO or nab-paclitaxel at $0,5,10$ or $20 \mathrm{nM}$ for $48 \mathrm{~h}$ in a humidified atmosphere containing $5 \% \mathrm{CO}_{2}$ at $37^{\circ} \mathrm{C}$. Cells were collected and stained with an Annexin V kit (cat no. 51-65874X; BD Biosciences) and a propidium iodide kit (cat no. 51-66211E; $\mathrm{BD}$ Biosciences) according to the manufacturer's protocol for $15 \mathrm{~min}$ at $25^{\circ} \mathrm{C}$ in the dark and then analyzed using a FACSCalibur machine. The data were analyzed using CellQuest software (version 2.0; BD Biosciences). Experiments were performed in triplicate, and data are expressed as mean \pm standard deviation.

Rat orthotopic tumor graft. A total of 20 adult 8-week old male Sprague-Dawley (SD) rats $(310 \pm 14$ g; BioLASCO Taiwan Co, Ltd, Taipei, Taiwan) were used for the experiments. Animals were divided equally $(n=5)$ into the following four groups: Control (Group 1), gemcitabine/oxaliplatin treatment (Group 2), paclitaxel treatment (Group 3) and nab-paclitaxel treatment (Group 4). The rats were housed in an animal facility room maintained in a $12 / 12 \mathrm{~h}$ light:dark cycle and at an ambient temperature of $22^{\circ} \mathrm{C}$. The animals had ad libitum access to food and water. The rats were administered with $300 \mathrm{mg} / \mathrm{l}$ thioacetamide (TAA) daily via drinking water for up to 20 weeks. All drug treatments administered to these animals were initiated in the 21 st week. The gemcitabine/oxaliplatin group received intraperitoneal injections of gemcitabine $(50 \mathrm{mg} / \mathrm{kg})$ and oxaliplatin $(2 \mathrm{mg} / \mathrm{kg})$ once every 2 weeks over a 4 -week period. Simultaneously, the paclitaxel group received an intravenous infusion of paclitaxel $(20 \mathrm{mg} / \mathrm{kg})$ for 5 consecutive days. Additionally, the nab-paclitaxel group received an intravenous slow infusion of nab-paclitaxel $(7.5 \mathrm{mg} / \mathrm{kg})$ for 5 consecutive days. The control group received intraperitoneal injections of phosphate buffered saline (PBS; pH 7.4) (Fig. 1).

Evaluation of treatment efficacy in rats by positron emission tomography. In order to evaluate the changes in glycolysis in live animals with liver tumors, 2-deoxy-2-[F-18] fluoro-d-glucose positron emission tomography $\left({ }^{18} \mathrm{~F}\right.$-FDG-PET) studies were performed at the Molecular Imaging Center of Chang Gung Memorial Hospital (Taoyuan, Taiwan). In brief, 20 rats were treated with TAA, prior to being subjected to serial PET scanning at 21, 23 and 25 weeks using the Inveon ${ }^{\mathrm{TM}}$ system (Siemens AG, Munich, Germany). Animals were assigned equally to the control and treatment groups based on their baseline PET results, to ensure similar PET-positive rates in the two groups. The details of radioligand preparation, scanning protocols and determination of optimal scanning time have been previously described by our group $(12,13)$. In brief, animals were fasted overnight prior to scanning and, $90 \mathrm{~min}$ after ${ }^{18} \mathrm{~F}$-FDG intravenous injection, 30-min static scans were obtained for all the animals. All imaging studies on animals were performed at $37^{\circ} \mathrm{C}$ under anesthesia $(2 \%$ isoflurane vaporized in $100 \%$ oxygen)-controlled imaging bed (Minerve System, Esternay, France). PET images were reconstructed using the 2-dimensional ordered subset expectation-maximization method (4 iterations and 16 subsets) without attenuation and scatter corrections. All imaging data were processed by the PMOD image analysis workstation (PMOD Technologies Ltd., Zurich, Switzerland). The largest liver tumor for each animal was identified by careful investigation of the three tumor image sets obtained for each rat. The uptake of ${ }^{18} \mathrm{~F}-\mathrm{FDG}$ by the normal and tumor liver tissues was quantified by calculating the standardized uptake value (SUV). The SUVs were calculated according to the recommendations of the European Organization for Research and Treatment of Cancer (14). Regions of interest (ROIs) in the tumors were determined by obtaining 


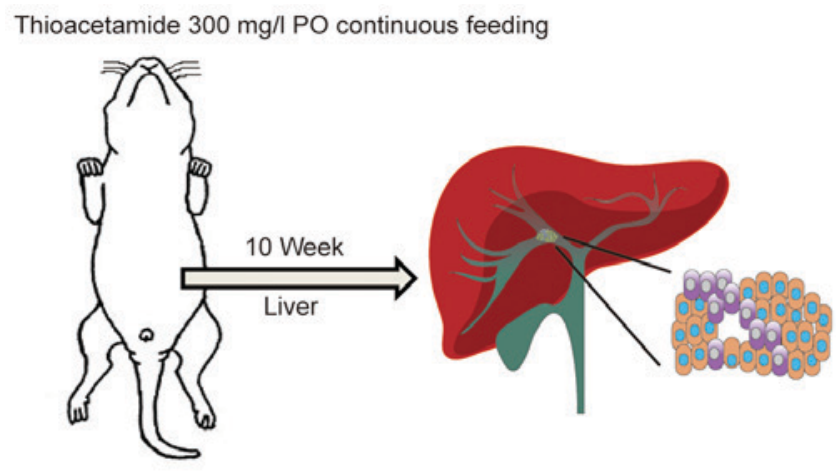

Normal cell

Cholangiolar proliferation with atypia

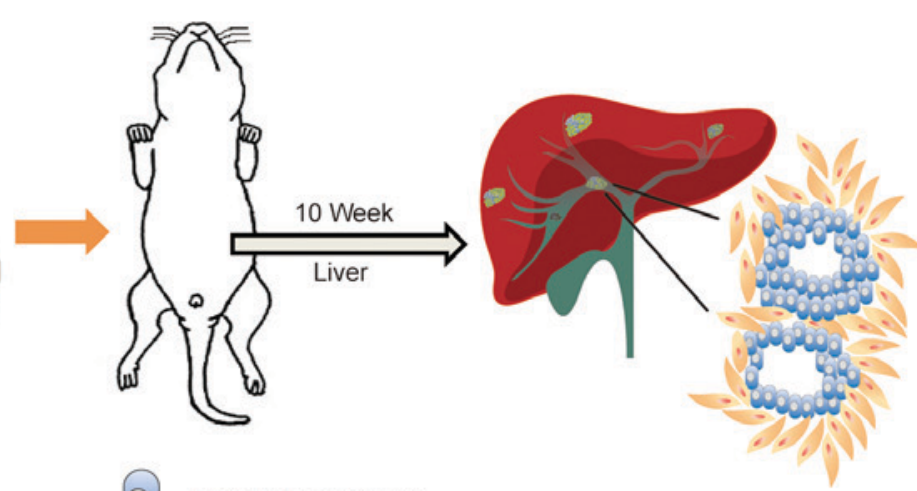

Cholangiocarcinoma

Cancer associated fibroblast

Figure 1. Spontaneous TAA-induced IH-CCA model. The schematic diagram illustrates the administration of TAA to rats for 16 weeks for the development of spontaneous IH-CCA. TAA (300 mg/l) was continuously administered to rats by oral gavage. After 10 weeks of administration, the animals were sacrificed and cholangiolar viability with atypical ductal cells was evaluated in the liver tissues. Continuous feeding of the rats with TAA for a further 6 weeks results in the development of cholangiocarcinoma with surrounding cancer-associated fibroblasts. TAA, thioacetamide; IH-CCA, intrahepatic cholangiocarcinoma.

transverse images of the selected tumors and measuring the largest diameter. The normal liver ROIs were also determined similarly by measuring the diameter of transverse images obtained from the normal liver tissues. The mean SUV ( $\mathrm{SUV}_{\text {mean }}$ ) of the normal and tumor liver tissues was calculated, and the respective tumor:liver $(\mathrm{T} / \mathrm{L})$ radioactivity ratio were compared for the two tissue types.

Immunofluorescence analysis for effects of drug treatment on rat tumor stroma. The study samples were obtained from rats treated with paclitaxel and nab-paclitaxel for 4 weeks. Formalin-fixed, paraffin-embedded slides (4- $\mu \mathrm{m}$ thickness) were prepared using a standard protocol. The slides were deparaffinized in 4 -min incubations in $100 \%$ xylene, then dehydrated in a graded alcohol serious of $100 \%$ for $5 \mathrm{~min} \times 2$, $95 \%$ for $5 \mathrm{~min}, 90 \%$ for $5 \mathrm{~min}, 70 \%$ for $5 \mathrm{~min}$ and distilled $\mathrm{H}_{2} \mathrm{O}$ for $5 \mathrm{~min} \times 3$ at $25^{\circ} \mathrm{C}$. Antigen retrieval took place using $\mathrm{pH} 6.0$ citric buffer at $121^{\circ} \mathrm{C}$ for $3 \mathrm{~min}$, washed 3 times in distilled water for $5 \mathrm{~min}$, then twice in Tris-buffered saline for $5 \mathrm{~min}$. Specimens were mounted using H33258 mounting solution and kept at $4^{\circ} \mathrm{C}$ until use. The slides were incubated with a primary antibody against $\alpha$-SMA (dilution, 1:200; cat. no. ab5694; Abcam, Cambridge, UK), a marker for activated fibroblasts, at $4^{\circ} \mathrm{C}$ overnight. The slides were washed with PBS with tween 20 following incubation with the primary antibody and were incubated at room temperature for $1 \mathrm{~h}$ with a goat anti-rabbit Alexa Fluor 568 (IgG H+L) secondary antibody (dilution, 1:200; cat. no. A11036; Molecular Probes; Thermo Fisher Scientific, Inc.). Nuclei were stained with Hoechst 33258 [mounted cover slide with $0.1 \mu \mathrm{g} / \mathrm{ml} \mathrm{H33258}$ mounting solution consisting of $0.1 \mu \mathrm{g} / \mathrm{ml} \mathrm{H} 33258$ (cat. no. H21491; Molecular Probes; Thermo Fisher Scientific, Inc.) in glycerol (cat no. G7757; Sigma-Aldrich; Merck KGaA) at $25^{\circ} \mathrm{C}$ and slides were then kept at $4^{\circ} \mathrm{C}$ until images were captured following three days]. Images were captured using the Leica TCS SP8X confocal system equipped with a LEICA DM6000 CS Microscope (objective lens 63x, subjective lens 10x, digital zoom 1x; final magnification, x630) (Leica Microsystems, Inc., Buffalo Grove, IL, USA).

Statistical analysis. The data are presented as the mean \pm standard deviation. SPSS software for Windows (Version 21.0; IBM Corp., Armonk, NY, USA) was used for statistical analysis. The SUV ratio between experimental and control animals was calculated using nonlinear regression analysis. Differences between the data from experimental and control animals were calculated and verified using the Mann-Whitney $\mathrm{U}$ test or the Kruskal-Wallis test. $\mathrm{P}<0.05$ was considered to indicate a statistically significant difference.

\section{Results}

Nab-paclitaxel and paclitaxel induced similar in vitro anti-proliferative effects. Human CCA KKU-100 and KKU-213 cell lines were treated with varying doses of paclitaxel and nab-paclitaxel. The MTT test indicated that the two compounds induced similar cytotoxicity in the different cell lines (paclitaxel $\mathrm{IC}_{50}$ values, $17.09 \mathrm{nM}$ for KKU-100 and $3.17 \mathrm{nM}$ for KKU-213; nab-paclitaxel $\mathrm{IC}_{50}$ values, $25.32 \mathrm{nM}$ for KKU-100 and $4.15 \mathrm{nM}$ for KKU-213; Fig. 2A). In order to confirm the apoptotic effect of nab-paclitaxel in CCA cells, the presence of apoptosis and the percentage of apoptotic cells were determined using a FITC Annexin V apoptosis detection kit. Nab-paclitaxel treatment increased the percentage of apoptotic cells within $48 \mathrm{~h}$ in a concentration-dependent manner; 54.32 and $60.78 \%$ of apoptotic KKU-100 and KKU-213 cells were observed following treatment with $20 \mathrm{nM}$ nab-paclitaxel (Fig. 2B).

\section{Nab-paclitaxel induced enhanced antitumor effects compared} with paclitaxel in a rat model of TAA-induced IH-CCA. Transverse, sagittal and coronal views of TAA-induced CCA tumors in control and drug-treated animals were evaluated by ${ }^{18}$ F-FDG-PET. Each group exhibited $>1$ FDG-avid tumor in 
A

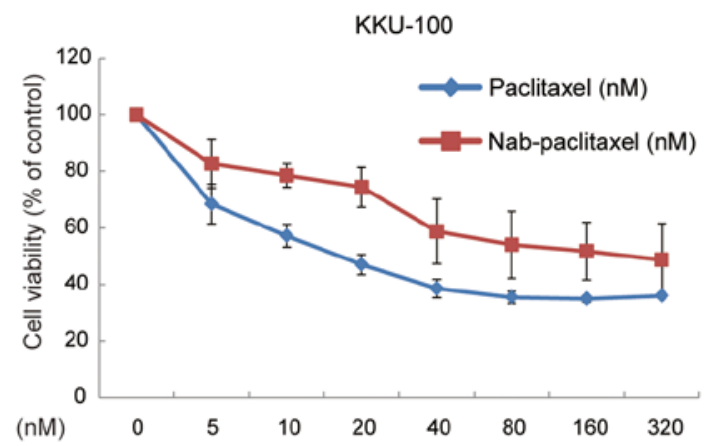

KKU-213

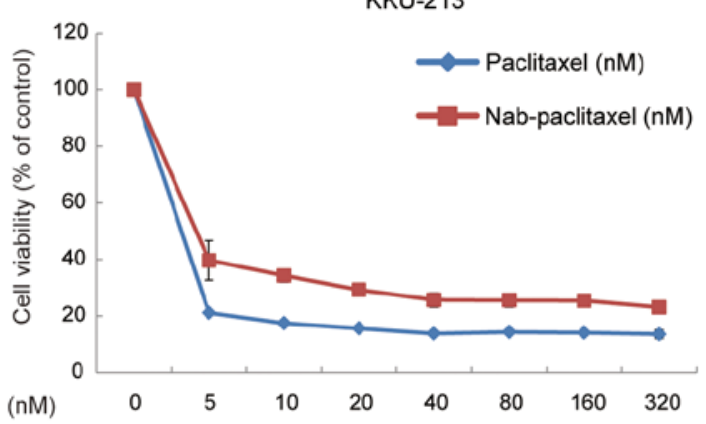

B

Nab-paclitaxel (nM)

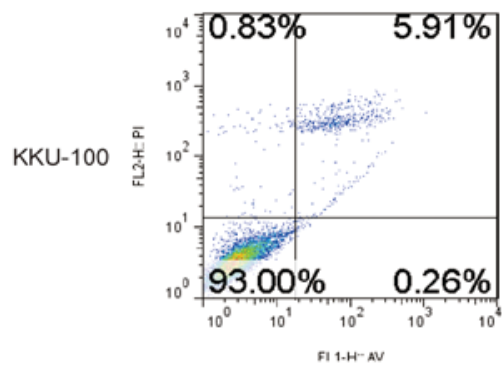

0

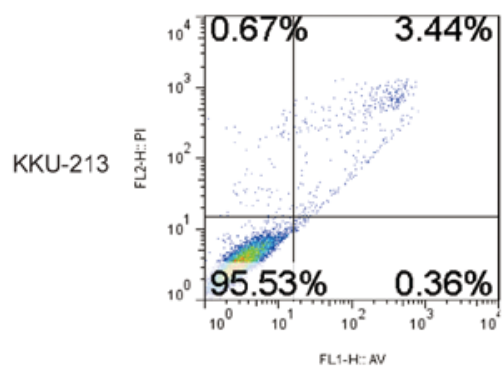

5
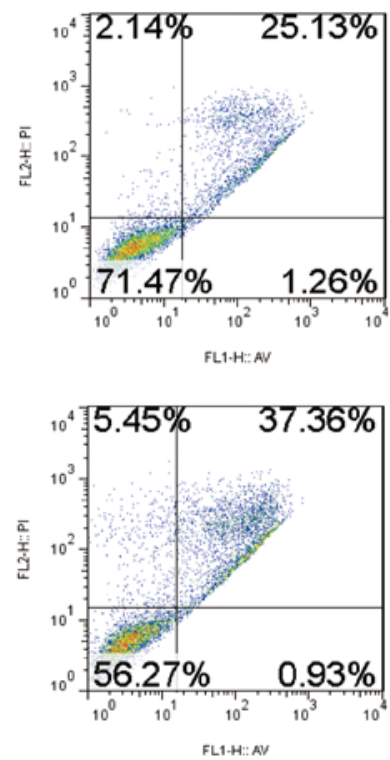

10
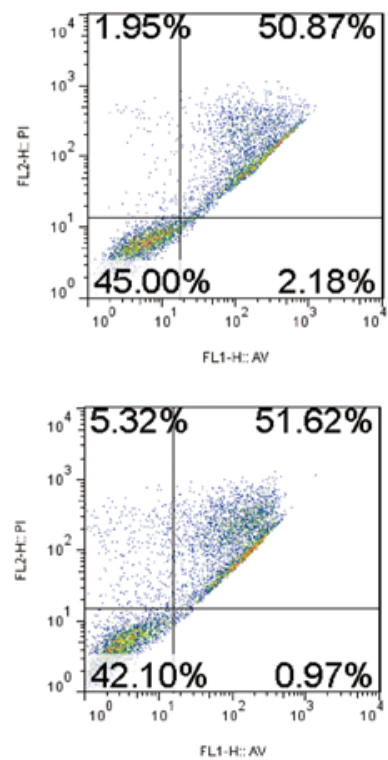

20
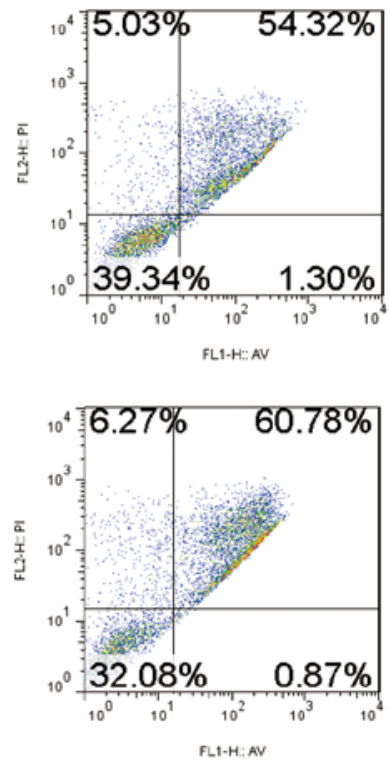
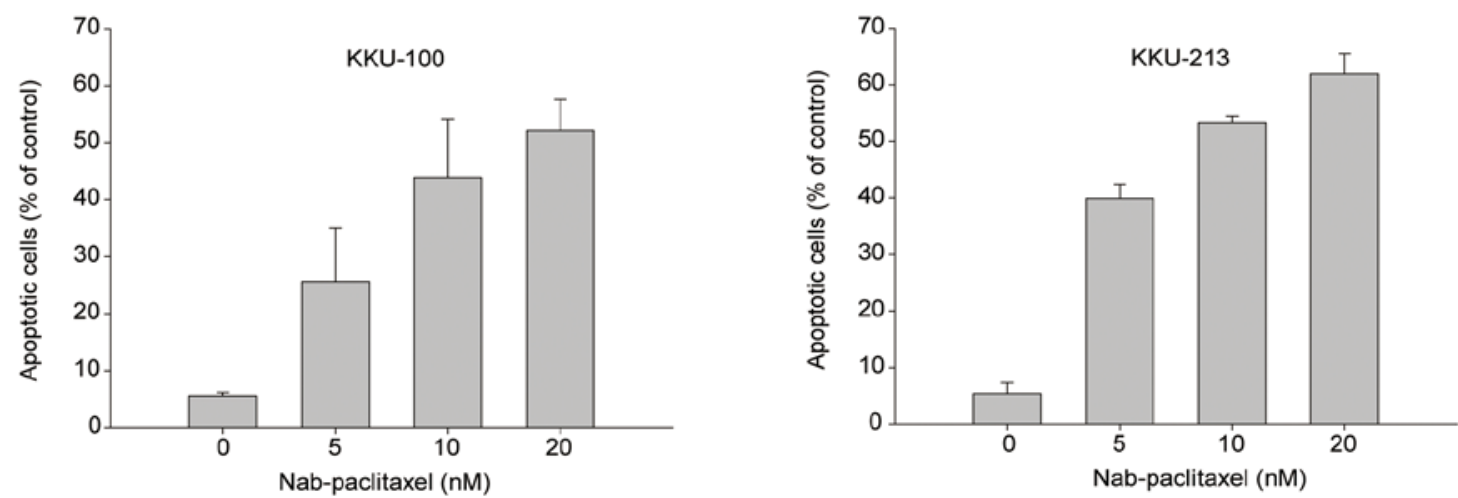

Figure 2. Nab-paclitaxel induced cholangiocarcinoma cell apoptosis and inhibited tumor cell viability. The antitumor effect of paclitaxel or nab-paclitaxel was observed in two CCA cell lines. (A) KKU-100 and KKU-213 cell lines were cultured with or without paclitaxel or nab-paclitaxel at indicated concentrations $(0,5,10,20,40,80,160$ or $320 \mathrm{nM})$ for $72 \mathrm{~h}$. Cell viability was evaluated using an MTT assay and data are presented as the mean \pm standard deviation of 3 independent experiments. (B) KKU-100 and KKU-213 cells were treated with or without nab-paclitaxel at indicated concentrations ( 0,5 , 10 or $20 \mathrm{nM}$ ) for $48 \mathrm{~h}$. Apoptotic cells were measured using the TACS Annexin V-FITC apoptosis detection kit and are represented as a percentage of the total cell death.

the liver after 20 weeks of TAA treatment, as demonstrated in the coronal view of the animal PET-CT (Fig. 3A). In the present study, nab-paclitaxel, paclitaxel or gemcitabine/oxaliplatin were administered to rats undergoing TAA treatment. The change in the T/L ratio of the SUV for each group is presented in Fig. 3B. The T/L ratio of the SUV exhibited steady elevation from the initial to the final scans in the control group (7.8-20.6\% elevation from 2 to 4 weeks post-experiment).
The T/L ratio of the SUV in the paclitaxel treatment group exhibited a mild increase until the final scans (5.6 and 5.1\% at 2 and 4 weeks post-experiment, respectively). By contrast, in the nab-paclitaxel and gemcitabine/oxaliplatin treatment groups, the T/L ratio of the SUV exhibited a decrease until the final scans (nab-paclitaxel, -28.5 and $-19.1 \%$, respectively; gemcitabine/oxaliplatin, -11.3 and $-5.4 \%$, respectively, at 2 and 4 weeks post-experiment). The results of the present 
A

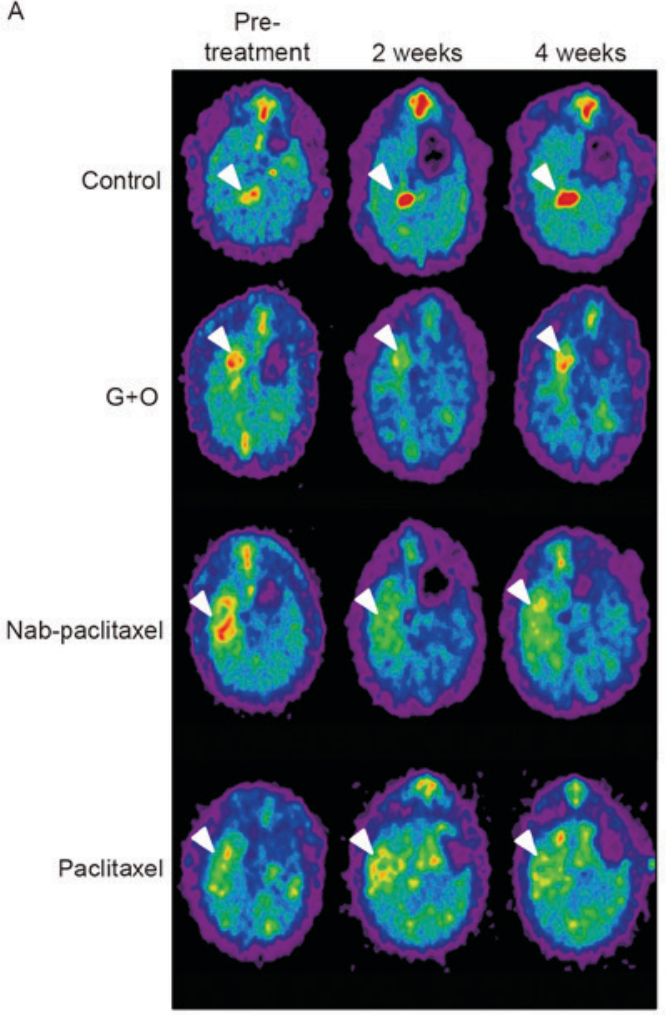

B

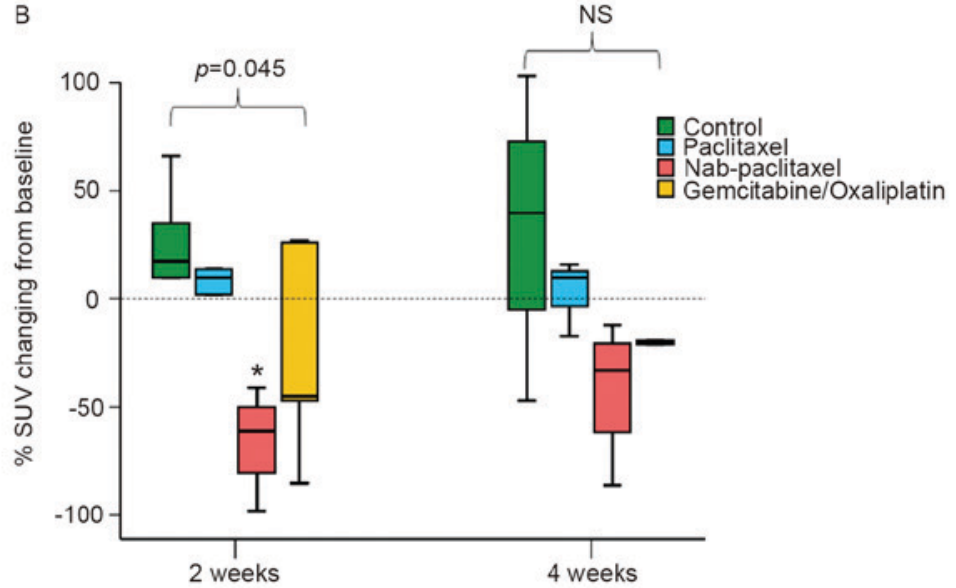

Figure 3. Nab-paclitaxel induced in vivo antitumor effects. (A) The spontaneous TAA-induced IH-CCA model was used for drug treatments. All pre-treatment groups of rats exhibited ${ }^{18}$ F-FDG-PET-detectable tumors following administration of TAA for 20 weeks. (B) Alterations in SUV 2 and 4 weeks post-treatment with paclitaxel, nab-paclitaxel and gemcitabine/oxaliplatin $(\mathrm{G}+\mathrm{O})$, respectively. ${ }^{*} \mathrm{P}<0.05$. TAA, thioacetamide; IH-CCA, intrahepatic cholangiocarcinoma; NS, not significant; SUV, standardized uptake value.

study indicated that treatment with nab-paclitaxel induced a significant decrease in the $\mathrm{T} / \mathrm{L}$ ratio of the $\mathrm{SUV}$, compared with the values reported for the control group $(\mathrm{P}<0.05$, control vs. group 3). Furthermore, nab-paclitaxel treatment resulted in significant suppression of in vivo tumor viability in the rat CCA model.

Nab-paclitaxel disrupts cancer-associated fibroblasts in rat $I H$-CCA. The immunofluorescence results for the resected IH-CCA of the rats are presented in Fig. 4. The red staining for $\alpha$-SMA was stronger and observed over the entire paclitaxel-treated tumor, compared with the weak, dispersed staining of the nab-paclitaxel-treated tumor (Fig. 4A). The confocal images in Fig. 4B indicated the anatomical association among the CAFs (with $\alpha$-SMA staining), vessels and IH-CCA, demonstrating that nab-paclitaxel broke the CAFs surrounding the IH-CCA.

\section{Discussion}

The results of the present study demonstrated that nab-paclitaxel may inhibit IH-CCA by disrupting the stromal CAFs. In an attempt to study this further, the TAA-induced IH-CCA rat model was selected for in vivo study. Multifocal bile ductal proliferation with marked atypia (15) was observed in the liver of the rats after 9 weeks of TAA administration, contrary to the observations made in a mouse xenograft model (15). Furthermore, 50\% of the rats developed IH-CCA with intense stromal desmoplasia from the 16th week (15).
From the 16-22th weeks, the incidence of invasive CCAs increased progressively in $100 \%$ of the rats (Fig. 1) (15). Therefore, this model served as a useful pre-clinical platform for developing therapeutic strategies for human CCA, including nab-paclitaxel $(12,13)$.

In the present study, paclitaxel and nab-paclitaxel induced similar anti-proliferative effects via induction of apoptosis in two human CCA cell lines (Fig. 2). However, only nab-paclitaxel induces in vivo antitumor effects in the TAA-induced rat model, demonstrated by a significant decrease in the T/L ratio of the SUV (Fig. 3). Paclitaxel did not induce any in vivo antitumor effects in the TAA-induced rat model. Furthermore, in the present study, the treatment effects induced by CAF on desmoplastic stroma were compared between the paclitaxel and the nab-paclitaxel groups. The disrupting effects on the stroma were found to be limited to the nab-paclitaxel group (Fig. 4). Therefore, the stromal disrupting effect may partially explain the effectiveness of nab-paclitaxel against IH-CCA. The current clinical observations support the aforementioned result, thereby providing an explanation for the ineffective use of paclitaxel in the treatment of biliary tract cancer since 1996 (16). However, Mucl and survivin have been suggested to be associated with the poor prognosis of IH-CCA (17). Furthermore, whether or not these two proteins changed following nab-paclitaxel treatment was examined. The immunohistochemistry stains of Muc1 and survivin exhibited no change prior to and following all treatments (data not shown), suggesting that nab-paclitaxel did not target these two proteins. 
A
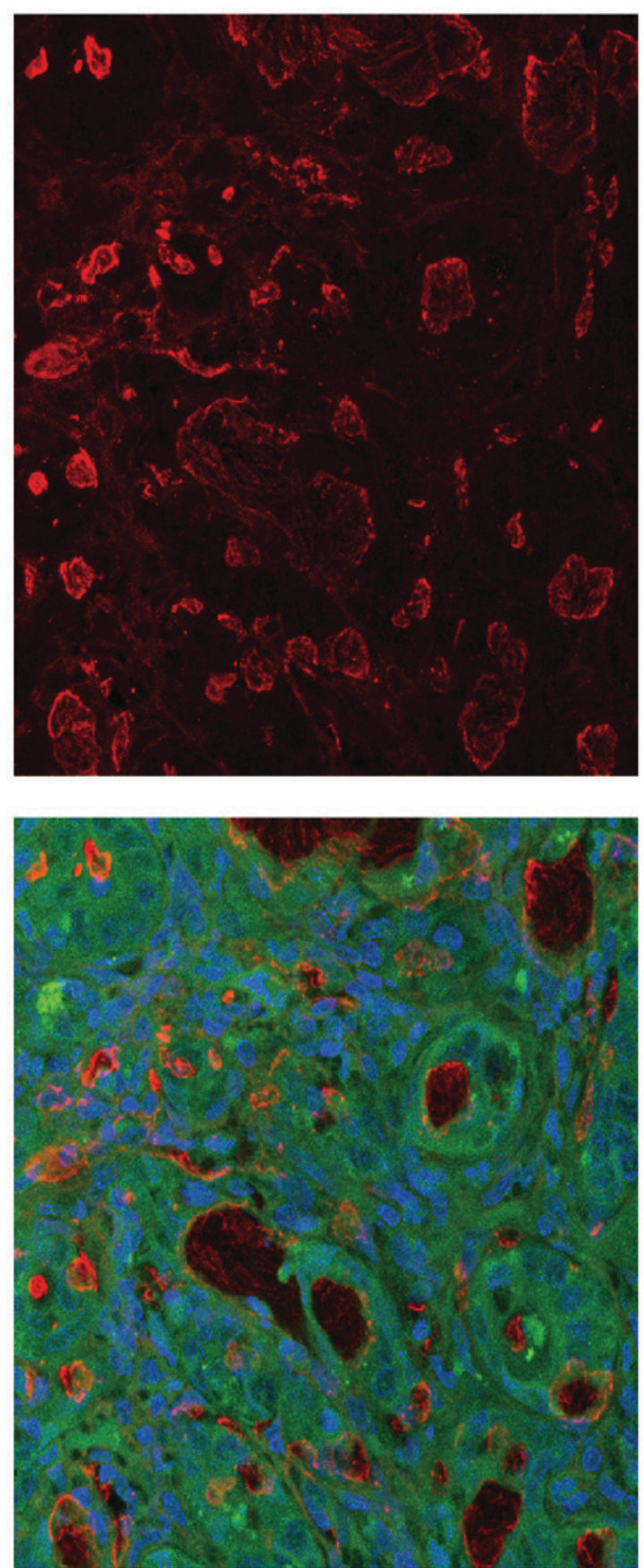

B
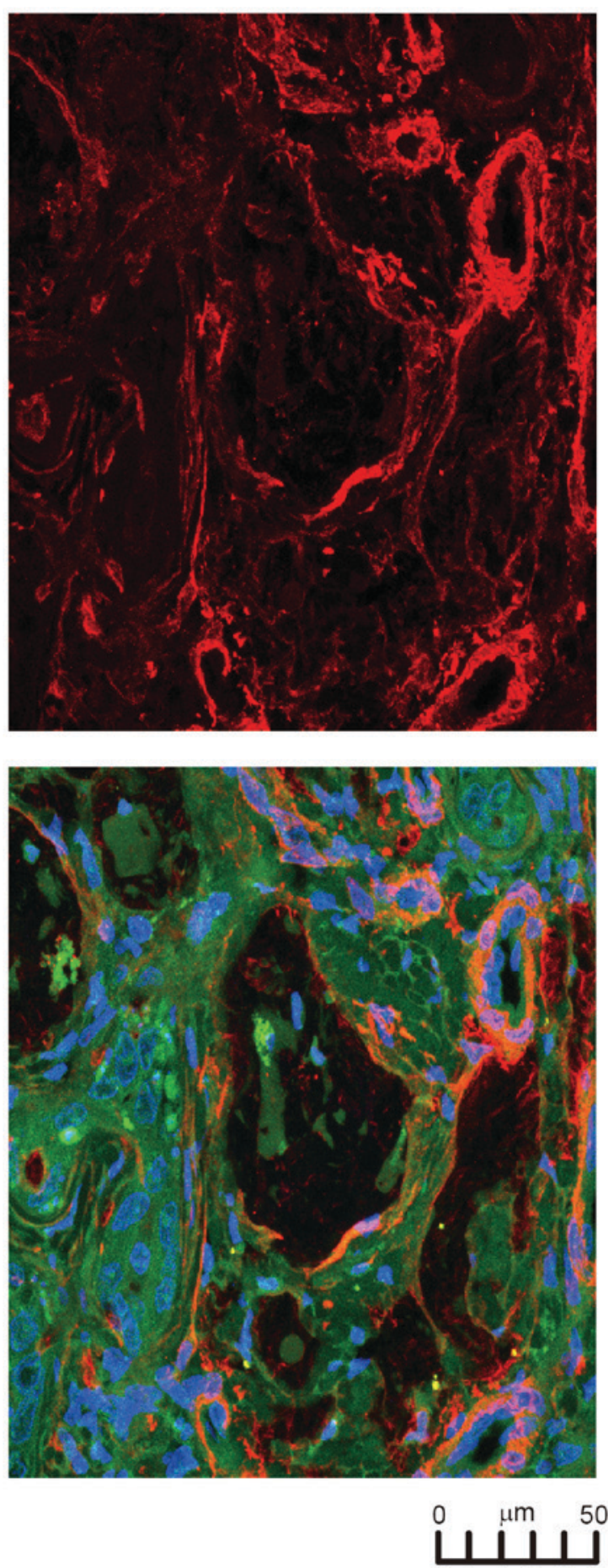

Figure 4. CAFs were disrupted by nab-paclitaxel in IH-CCA. (A) In order to study the effects of the treatments on CAFs, tissues from rats treated with the indicated agents were stained with $\alpha$-SMA (red) and quantitated for the presence of activated and total fibroblasts. (B) The confocal microscopic analysis of $\alpha$-SMA (red) and 4'6-diamidino-2-phenylindole (blue) immunofluorescence staining of total and activated CAFs in tumors treated with paclitaxel and nab-paclitaxel. CAFs, cancer-associated fibroblasts; IH-CCA, intrahepatic cholangiocarcinoma; $\alpha$-SMA, $\alpha$-smooth muscle actin.

There are certain limitations to the present study. To begin with, although the present study investigates an orthotopic CCA with desmoplastic stroma originating from the rat liver, such induced CCA may not completely simulate the conditions observed in human CCA. However, this model overcomes the drawbacks of a xenograft CCA model that lacks surrounding CAFs. Secondly, the small number of rats used in the four different treatment groups may lower the statistical power of the present study. For example, the animal PET revealed the significant inhibitory effect of nab-paclitaxel compared with that of paclitaxel, but the immunofluorescence study was unable to statistically confirm these observations.

In conclusion, the results of the present study demonstrated that nab-paclitaxel is effective against IH-CCA via disruption of the surrounding CAFs. Since there are few treatment options 
available for IH-CCA, this result may provide useful information in designing clinical trials, as well as for the identification of biomarker in the future.

\section{Acknowledgements}

The authors would like to thank Miss Meng-Lun Lu (Taipei Veterans General Hospital, Tapei, Taiwan) for her technology support.

\section{Funding}

The work was supported by Taiwan Cancer Clinic Foundation and the Szu-Yuan Research Foundation of International Medicine. Additional support was provided by the Taipei Veterans General Hospital (grant nos. V105C-057 and V104E16-003-MY3-2 to Ming-Huang Chen), the Chang Gung Memorial Hospital (grant nos. NMRPG5D6032, CMRPG3E1611, CMRPG3E1612, CRRPG3F0031 and NMRPG3F6021 to Chun-Nan Yeh), and the Ministry of Science and Technology (grant nos. MOST104-2314-B-075-064-MY2 to Ming-Huang Chen, MOST103-2314-B-182A-081-MY2 and MOST105-2314-B-182A-041-MY2 to Chun-Nan Yeh).

\section{Availability of data and materials}

All data generated or analyzed during this study are included in this published article.

\section{Authors' contributions}

PMC analyzed the data and wrote the manuscript. CTC, RCW, $\mathrm{KCC}, \mathrm{CYL}$ and $\mathrm{MHaC}$ contributed to study design and data collection. YHC, TSY helped with the animal experiments. $\mathrm{MHuC}$ helped cell experiments. CNY guided the animal model and associated tissue IHC staining analysis. All authors read and approved the final manuscript.

\section{Ethics approval and consent to participate}

The present study was approved by the Institutional Animal Care and Use Committee of Chang Gung Memorial Hospital (Taoyuan, Taiwan).

\section{Consent for publication}

Not applicable.

\section{Competing interests}

The authors declare that they have no competing interests.

\section{Reference}

1. Ustundag Y and Bayraktar Y: Cholangiocarcinoma: A compact review of the literature. World J Gastroenterol 14: 6458-6466, 2008.

2. Khan SA, Thomas HC, Davidson BR and Taylor-Robinson SD: Cholangiocarcinoma. Lancet 366: 1303-1314, 2005.

3. Patel T: Increasing incidence and mortality of primary intrahepatic cholangiocarcinoma in the United States. Hepatology 33: 1353-1357, 2001

4. Valle J, Wasan H, Palmer DH, Cunningham D, Anthoney A, Maraveyas A, Madhusudan S, Iveson T, Hughes S, Pereira SP, et al: Cisplatin plus gemcitabine versus gemcitabine for biliary tract cancer. N Engl J Med 362: 1273-1281, 2010.

5. Hezel AF, Deshpande V and Zhu AX: Genetics of biliary tract cancers and emerging targeted therapies. J Clin Oncol 28: 3531-3540, 2010.

6. Zhu AX and Hezel AF: Development of molecularly targeted therapies in biliary tract cancers: Reassessing the challenges and opportunities. Hepatology 53: 695-704, 2011.

7. Sirica AE and Gores GJ: Desmoplastic stroma and cholangiocarcinoma: Clinical implications and therapeutic targeting. Hepatology 59: 2397-2402, 2014.

8. Sirica AE: The role of cancer-associated myofibroblasts in intrahepatic cholangiocarcinoma. Nat Rev Gastroenterol Hepatol 9: 44-54, 2011.

9. DeClerck YA: Desmoplasia: A response or a niche? Cancer Discov 2: 772-724, 2012.

10. Alvarez R, Musteanu M, Garcia-Garcia E, Lopez-Casas PP, Megias D, Guerra C, Muñoz M, Quijano Y, Cubillo A, Rodriguez-Pascual J, et al: Stromal disrupting effects of nab-paclitaxel in pancreatic cancer. Br J Cancer 109: 926-933, 2013.

11. Von Hoff DD, Ervin T, Arena FP, Chiorean EG, Infante J, Moore M, Seay T, Tjulandin SA, Ma WW, Saleh MN, et al: Increased survival in pancreatic cancer with nab-paclitaxel plus gemcitabine. N Engl J Med 369: 1691-1703, 2013.

12. Chen MH, Lin KJ, Yang WL, Kao YW, Chen TW, Chao SC, Chang PM, Liu CY, Tzeng CH, Chao Y, et al: Gene expression-based chemical genomics identifies heat-shock protein 90 inhibitors as potential therapeutic drugs in cholangiocarcinoma. Cancer 119: 293-303, 2013.

13. Chen MH, Chiang KC, Cheng CT, Huang SC, Chen YY, Chen TW, Yeh TS, Jan YY, Wang HM, Weng JJ, et al: Antitumor activity of the combination of an HSP90 inhibitor and a PI3K/mTOR dual inhibitor against cholangiocarcinoma. Oncotarget 5: 2372-2389, 2014.

14. Young H, Baum R, Cremerius U, Herholz K, Hoekstra O, Lammertsma AA, Pruim J and Price P: Measurement of clinical and subclinical tumour response using [18F]-fluorodeoxyglucose and positron emission tomography: Review and 1999 EORTC recommendations. European organization for research and treatment of cancer (EORTC) PET study group. Eur J Cancer 35: 1773-1782, 1999.

15. Yeh CN, Maitra A, Lee KF, Jan YY and Chen MF: Thioacetamide-induced intestinal-type cholangiocarcinoma in rat: An animal model recapitulating the multi-stage progression of human cholangiocarcinoma. Carcinogenesis 25: 631-636, 2004.

16. Jones DV Jr, Lozano R, Hoque A, Markowitz A and Patt YZ: Phase II study of paclitaxel therapy for unresectable biliary tree carcinomas. J Clin Oncol 14: 2306-2310, 1996.

17. Sirica AE, Dumur CI, Campbell DJ, Almenara JA, Ogunwobi OO and Dewitt JL: Intrahepatic cholangiocarcinoma progression: Prognostic factors and basic mechanisms. Clin Gastroenterol Hepatol 7 (11 Suppl): S68-S78, 2009. 